\title{
Evaluation of Lead Exposure by Hand Wipes: A Review of the Effectiveness of Personal Hygiene on Industrial Sites
}

\author{
Kevin Guth, Marie Bourgeois, Giffe Johnson, Raymond Harbison \\ Center for Environmental and Occupational Risk Analysis and Management, College of Public Health, University of South \\ Florida, Tampa, FL, USA \\ Email: rharbiso@health.usf.edu
}

How to cite this paper: Guth, K., Bourgeois, M., Johnson, G. and Harbison, R. (2019) Evaluation of Lead Exposure by Hand Wipes: A Review of the Effectiveness of Personal Hygiene on Industrial Sites. Occupational Diseases and Environmental Medicine, 7, 135-143.

https://doi.org/10.4236/odem.2019.74011

Received: August 20, 2019

Accepted: October 8, 2019

Published: October 11, 2019

Copyright $\odot 2019$ by author(s) and Scientific Research Publishing Inc. This work is licensed under the Creative Commons Attribution International License (CC BY 4.0).

http://creativecommons.org/licenses/by/4.0/

\begin{abstract}
To evaluate the effectiveness of personal hygiene (handwashing) amongst workers at industrial sites to remove lead from their hands, a retrospective analysis of hand wipe samples was conducted using data collected by two contractors from two bridge painting projects for total lead using method ASTM E-1979-17/EPA SW846 7000B. Exposures resulted from the removal of lead-based paint from the structure and trace elements of lead found in the abrasive blast media. In total, six work tasks were evaluated and sixty unique hand wipe samples were evaluated. Thirty samples were collected during the worker's lunch break, after they had reportedly washed their hands with a further 30 collected at the end of the workday following the same protocol. To be included in this evaluation, the contractors were required to follow NIOSH Method 9105 (Lead in Dust Wipes-Dermal Surfaces) with subsequent analysis of samples for total lead by an American Industrial Hygiene Association (AIHA) accredited laboratory. All 60 samples contained detectable lead. The lead exposures ranged from $19.5 \mu \mathrm{g}$ to $3420 \mu \mathrm{g}$. The geometric mean for the samples collected was $337 \mu$ g. These results indicate that current personal hygiene practices at the evaluated sites are not effective at removing lead from worker's hands during and after the workday. They also suggest that the residual lead measured on the workers' hands, at the end of the shift, is likely contributing to the elevated blood lead levels in this population.
\end{abstract}

\section{Keywords}

OSHA, Lead Exposure Assessment, Painter, Blood Lead Level, Prevention and Control, Industrial Contractor 


\section{Introduction}

It has been 26 years since the passage of the OSHA lead in construction standard [1] intended to control both inhalation and ingestion exposures. Despite advances in engineering controls and work practices, elevated blood lead levels (BLLs $>25 \mu \mathrm{g} / \mathrm{dl}$ ) persist among workers within the industrial painting sector [2]. Given the poor health outcomes associated with the uptake of lead in humans [3], it is vital to understand why many workers exposed to lead in the construction industry have BLLs trending upwards [4] [5]. OSHA's construction lead standard [1] requires the employment of control methods to reduce employee exposures to inorganic lead for the primary exposure pathways, inhalation and ingestion. Unlike the inhalation route of exposure that is controlled by a legally enforceable Permissible Exposure Limit (PEL), there is no dermal loading exposure limit provided by OSHA in the lead in construction standard [1] for managing ingestion exposures. As a result, very little is known about the intensity of worker lead dermal loading and the effectiveness of handwashing to remove lead from a worker's hands after exposure. Lead dermal loading presents a health risk because of the potential for worker ingestion due to hand-to-mouth transfer [6]. To minimize lead ingestion exposures, a contractor must institute a control measure. Handwashing with soap and water before a worker eats, drinks or smokes is the principal exposure control method for reducing lead ingestion exposure [1].

The literature related to assessments of the efficacy of personal hygiene (handwashing) for workers exposed to lead is sparse, most likely due to the lack of a regulatory emphasis on this route of exposure. Some researchers have measured the efficacy of work practice controls (personal protective equipment (PPE) \& handwashing) and their impact on the elevated blood lead levels. For instance, Rodrigues et al. [7] and Askin \& Volkmann [8] both found an association between lead dermal loading on the hands of workers and elevated blood lead levels. Several studies have also provided evidence to support the proper use of PPE and basic personal hygiene practices in having a measurable impact upon the uptake of lead among workers [7] [9] [10]. There is also a growing body of evidence related to residual lead levels on the hands of workers after washing them at the conclusion of the workday [7] [8] [11] [12].

The purpose of this study is to evaluate the effectiveness of personal hygiene (handwashing) amongst workers at industrial sites to remove lead from their hands. The results of this study provide much-needed information on the efficacy of handwashing as an exposure control measure that can help painting contractors refine their personal hygiene practices to minimize worker exposure.

\section{Methods}

\subsection{Study Population}

Retrospective hand wipe data were obtained from two industrial painting contractors who assessed lead worker exposure by collecting hand wipe samples 
from workers engaged in short-term bridge painting projects for risk management purposes. Both painting contractors had a work crew that consisted of 15 workers. The tasks that each worker undertook are detailed within Table 4 of the results. The sources of lead exposure by job task are detailed in Table 1 .

Each contractor reported the existing coating that was removed by abrasive blasting contained lead in the dry paint film. Both contracting firms used a slag (coal) bulk abrasive blast media during each hand wipe exposure assessment; analyses of this media also indicated trace amounts of lead.

\subsection{Hand Wipe Sample Collection Method and Analysis}

The contractors used Lead WipeTM sampling media for the collection of the wipe samples. This sample media meets the ASTM E1792 requirements for collection and subsequent analysis of lead wipe samples as required by the EPA [13]. The analytical laboratory (Environmental Hazards Services, LLC Richmond, Virginia) provided the hand wipe sample media. The contractor's representative put on a clean pair of latex gloves before handling each sample to prevent cross-contamination. Each wipe sample was placed into a pre-cleaned for metals $(50 \mathrm{ml})$ centrifuge tube with a locking cap. The contractor submitted field blanks to the laboratory to ensure the handling, storage or shipment of samples was not the lead source measured on the sample media. The contractors selected for this study were required to follow NIOSH Method 9105, Lead in Dust Wipes for Dermal Surfaces [14], to ensure similar accuracy and precision in the sample collection method. The industrial contractors obtained wipe samples of their employee's hands after exposure to lead at mid shift $(\mathrm{N}=30)$ before allowing employees to eat or drink, and at the conclusion of the workday $(\mathrm{N}=30)$ resulting in 60 total hand wipe samples available for evaluation. Environmental Hazards Services analyzed the hand wipe samples and the field blanks in accordance with ASTM-E-1979/EPA SW846 7000B.

Table 1. Work process and lead exposure by job task.

\begin{tabular}{cc}
\hline Work Process & Exposure by Job Task \\
\hline $\begin{array}{c}\text { Work platform and } \\
\text { containment tarpaulins installation } \\
\text { Abrasive Blasting }\end{array}$ & Abrasive Blaster \\
$\begin{array}{c}\text { Inspection of the steel structure after the } \\
\text { abrasive blasting and before painting operations }\end{array}$ & Quality Control Inspector, Quality control \\
Vacuuming of spent abrasive \\
during and after abrasive blasting \\
Painting and inspection of the \\
paint system after it is applied \\
Sismantling of containment and platform
\end{tabular}




\subsection{Exposure Measurement (Dermal Loading)}

Dermal loading for this evaluation is defined as the lead concentration measured on the total surface area of workers' hands by a single ASTM E1792 hand wipe. Traditional industrial hygiene practice involves the evaluation of the workplace by obtaining field measurements and comparing the exposure concentrations to established occupational exposure limits (OELs). The exposure measurement is often compared to an American Industrial Hygiene Association (AIHA) rating guideline [15] to measure the efficacy of the controls in place. Measuring the effectiveness of handwashing as an exposure control is hampered by the lack of an established inorganic lead dermal OEL as a basis for comparison. To address this shortcoming, a modified dermal exposure screening approach was used to classify the lead dermal loading on worker's hands, as illustrated below in Table 2 [16]. The classification system consisted of three dermal loading exposure tiers (low, medium, high). The tiers of dermal loading do not represent safe or unsafe concentrations of lead; instead, they were used to assess how effective the control (handwashing) is at reducing lead from the workers' hands.

Adapted from Energy \& Building Research Center (EBRC), Kuwait Institute for Scientific Research (August 2007). Human Health Risk Assessment Guidance for Metal: Fact Sheet No. 1.

\subsection{Handwashing Evaluation Decision Logic}

After the collection and analysis of the handwipe samples, the geometric mean lead exposure for each work classification was compared to the dermal loading tiers using the decision rules established below to assess the effectiveness of the contractor's personal hygiene program and to determine what if any corrective action was necessary (Table 3 ).

\subsection{Data Analysis}

The data were analyzed by using the American Industrial Hygiene Association's Multilingual IHSTAT + MS Excel application [17]. This application is used to compare the measured industrial hygiene exposure data to an OEL to properly characterize the exposure. However, as there is no OEL for lead dermal loading, the upper bound of the Tier 1 dermal loading classification $(5 \mu \mathrm{g})$ was used to interpret the wipe sampling data. A Shapiro-Wilk test of the exposure data was conducted and found that the mid-shift and end-of-the shift data sets for all classifications were log-normally distributed.

Table 2. Dermal loading (hands)-exposure tiers.

\begin{tabular}{ccc}
\hline Tier & $(\mu \mathrm{g} \mathrm{Pb} /$ hand wipe $)$ & Dermal Loading \\
\hline 1 & $0-5$ & Low \\
2 & $6-50$ & Medium \\
3 & $>50$ & High \\
\hline
\end{tabular}


Table 3. Evaluation decision logic.

\begin{tabular}{|c|c|}
\hline Decision Rule & Decision/Action \\
\hline $\begin{array}{l}\text { 1. If the geometric mean lead } \\
\text { concentration is }<6 \mu \mathrm{g} / \text { handwipe }\end{array}$ & $\begin{array}{l}\text { Conclude that the handwashing program is effective } \\
\text { at reducing ingestion exposure. } \\
\text { No further action is required. }\end{array}$ \\
\hline $\begin{array}{l}\text { 2. If the geometric mean lead } \\
\text { concentration is } \geq 6 \mu \mathrm{g} / \mathrm{handwipe} \\
\text { and } \leq 50 \mu \mathrm{g} / \mathrm{handwipe}\end{array}$ & $\begin{array}{l}\text { Conclude the handwashing program needs improvement. } \\
\text { An evaluation of the program to see what steps can be } \\
\text { taken to improve handwashing practices is necessary. } \\
\text { Conduct training centered on effective handwashing } \\
\text { practices for all affected employees. Follow up wipe } \\
\text { sampling should be conducted after a training session } \\
\text { has been held. }\end{array}$ \\
\hline $\begin{array}{l}\text { 3. If the geometric mean lead } \\
\text { concentration is }>50 \mu \mathrm{g} / \text { handwipe }\end{array}$ & $\begin{array}{l}\text { Conclude that the handwashing program is not effective at } \\
\text { preventing lead exposures through the ingestion pathway. } \\
\text { A thorough evaluation of the program is warranted to } \\
\text { determine the root causes of the high dermal loading. } \\
\text { Conduct training centered on effective handwashing } \\
\text { practices for all affected employees. Follow up wipe sampling } \\
\text { should be conducted after a training session has been held. }\end{array}$ \\
\hline
\end{tabular}

The geometric mean for classifying dermal exposure loading was selected for two reasons: 1) the geometric mean is the most appropriate metric in this case for characterizing typical exposures levels [18]; and, 2) previous research on lead dermal loading evaluated by hand wipe sampling employed this same measure for assessing the effect of dermal exposure on internal dose [7].

\section{Results and Discussion}

\subsection{Results}

In total, 30 workers were included in the study. Table 4 details the number of workers in each project, and the tasks they undertook, by project site.

The geometric mean (GM) lead exposures and geometric standard deviations (GSD) for all work tasks evaluated by hand wipes are summarized below in Table 5. There were elevated levels of lead measured on workers' hands for every evaluated work task at the conclusion of the workday after handwashing. All 60 hand wipe samples contained detectable lead. The concentration of lead measured on the worker's hands for this study ranged from $19.5 \mu \mathrm{g}$ to $3420 \mu \mathrm{g}$. There was no reportable lead measured in the wipe sample field blanks. For all work tasks combined, when comparing the geometric means, there was a $10 \%$ reduction of lead dermal (hand) loading from the mid-shift to the end of shift. The painters experienced the most intense lead exposure, but they also had the greatest reduction in lead levels between the mid-shift and the end of shift.

Exposures were greater at the end of the shift in two of the six work groups evaluated. Inspectors had the largest differential at 4.9 times greater lead concentration at the end of shift dermal exposure compared with the mid-shift exposure. 
Table 4. Job tasks evaluated.

\begin{tabular}{ccc}
\hline Job Task & Project $1(\mathrm{n}=\mathbf{1 5})$ & Project 2 $(\mathrm{n}=\mathbf{1 5})$ \\
\hline Abrasive Blaster & 8 & 9 \\
Painter & 3 & 3 \\
Inspector/Paint Inspector & 1 & 1 \\
Superintendent & 1 & 1 \\
Equipment Operator Quality Control & 1 & 0 \\
Supervisor & 1 & 1 \\
\hline
\end{tabular}

Table 5. Dermal (hand) lead exposures.

\begin{tabular}{ccccccc}
\hline \multicolumn{7}{c}{ Dermal Exposure (ug/hand wipe) N $=60$} \\
Work Tasks & GM & GSD & Min & Max & Tier & Samples \\
\hline All (Mid-Shift) & 342.0 & 3.5 & 24.6 & 3420 & 3 & 30 \\
All (End-Shift & 306.8 & 4.0 & 19.5 & 2400 & 3 & 30 \\
Abrasive Blasters (Mid-Shift) & 363.0 & 2.5 & 51.5 & 2450 & 3 & 17 \\
Abrasive Blasters (End-Shift) & 353.5 & 3.6 & 25.7 & 1570 & 3 & 17 \\
Painters (Mid-Shift) & 900.1 & 3.0 & 164 & 3420 & 3 & 6 \\
Painters (End-Shift) & 476.3 & 3.7 & 78 & 2400 & 3 & 6 \\
QC Supervisor (Mid Shift) & 53.2 & 1.5 & 39.4 & 71.9 & 3 & 2 \\
QC Supervisor (End Shift) & 21.4 & 1.1 & 19.5 & 23.4 & 2 & 2 \\
Inspector (Mid Shift) & 37.0 & 1.8 & 24.6 & 55.7 & 2 & 2 \\
Inspector (End Shift) & 183.6 & 4.3 & 65.2 & 517 & 3 & 2 \\
Superintendent (Mid Shift) & 445.4 & 4.4 & 155 & 1280 & 3 & 2 \\
Superintendent (End Shift) & 588.6 & 3.9 & 225 & 1540 & 3 & 2 \\
Equipment Operator (Mid Shift) & - & - & - & 771 & - & 1 \\
Equipment Operator (End Shift) & - & & & 308 & - & 1 \\
\hline
\end{tabular}

Note: $\mathrm{GM}=$ Geometric Mean, GSD = Geometric Standard Deviation.

\subsection{Discussion}

The current study sought to use hand wipe sampling data collected by contractors from two projects to evaluate lead levels on workers' hands in the industrial painting sector after handwashing. Exposures evaluated in the all work tasks category were classified as Tier 3 for mid shift and the end of the shift. These exposure results represent the upper bound of dermal loading on worker's hands suggesting that the evaluated hand washing programs are not effective at controlling ingestion lead exposures. If this retrospective data is representative of the industrial painting industry, it is likely poor personal hygiene is contributing to the worker's lead body burden, which in turn may explain why elevated blood lead levels have remained constant in the target population. The results of this study support the findings of many other researchers who also found residual 
lead on workers' hands after handwashing at the conclusion of the workday [7] [8] [11] [12]. Furthermore, the findings of this study support the need for improved work practice controls to manage lead exposures.

These results have several associated limitations. The lead removal efficacy of the hand wipe sampling method used in this study is unknown. Previous research by Boeniger [19] found less than $76 \%$ collection efficiency for lead using four consecutive dermal wipes as the exposure assessment method. Worker exposures are likely greater than what were measured during this sampling campaign. Likewise, the lead removal efficacy of the soap used for worker handwashing during this study is also unknown. Esswein \& Boeniger [20] found that standard soap and water does a poor job of removing lead from the skin. This is a plausible reason why residual lead has been found in several studies on workers' hands after handwashing.

Another limitation of this study is the lead concentration in the paint and in the abrasive blasting media was not reported. Other work sites could have a higher lead concentration in the existing coating system and the abrasive blast media resulting in potential higher dermal loading.

We used a non-parametric decision logic adapted from Hewett [21] for evaluating the contractor's handwashing program, conservatively classifying exposure measurements in tier 3 as representative of an ineffective exposure control and the tier 2 exposures as representative of an exposure control that needs improvement to account for the likely underestimation of exposure due to the poor collection efficiency associated with the dermal wipe sampling method.

A hand dermal loading exposure limit would be useful to measure compliance with existing lead paint removal written program requirements and (indirectly) more effectively manage ingestion exposures. However, the probability that OSHA will develop an OEL for hand dermal loading for lead or any other chemical agent is low due to toxicological quantification limitations [22].

Additional research is warranted based on these results. Active hand wipe sampling and observations of employer instituted work practice controls on a lead paint removal project with a tiered dermal loading exposure limit approach could provide many valuable insights into effective ways to reduce lead ingestion exposures.

\section{Conclusions}

The results of the current study indicate that residual lead dust remained on worker's hands even after handwashing. This is troubling, as elevated dermal lead loading represents serious health consequences for the exposed employees. Contractors might seek to re-evaluate their personal protective equipment hazard evaluation processes to include dermal loading as a significant risk factor for lead exposure and thus monitor and control ingestion exposure.

These results will be useful in raising awareness in the industrial painting industry that personal hygiene practices, and broader practices which protect 
against lead exposure, must be improved to prevent the uptake of lead during the removal of lead-based paint.

\section{Acknowledgements}

This work was partially supported by the Center for Environmental and Occupational Risk Analysis and Management at the University of South Florida.

\section{Conflicts of Interest}

The authors declare no conflicts of interest regarding the publication of this paper.

\section{References}

[1] Occupational Safety and Health Administration (OSHA), U.S. Department of Labor (1993) 29 CFR 1926: Lead Exposure in Construction; Interim Final Rule. Washington DC.

[2] OSHA (2008) Instruction CPL 03-00-009; National Emphasis Program: Lead.

[3] Agency for Toxic Substances and Disease Registry (ATSDR) (2007) Toxicological Profile for Lead. https://www.atsdr.cdc.gov/toxprofiles/tp.asp?id=96\&tid=22

[4] Alarcon, W.A. (2015) Elevated Blood Lead Levels among Employed Adults-United States, 1994-2012. Morbidity and Mortality Weekly Report (MMWR), 62, 52-75. https://www.cdc.gov/mmwr/preview/mmwrhtml/mm6254a4.htm https://doi.org/10.15585/mmwr.mm6254a4

[5] CDC, Adult Blood Lead Epidemiology and Surveillance (ABLES), NIOSH Workplace Safety and Health Topic (2019). https://www.cdc.gov/niosh/topics/ables/data.html

[6] Sahmel, J., Hsu, E.I., Avens, H.J., Beckett, E.M. and Devlin, K.D. (2015) Estimation of Hand-to-Mouth Transfer Efficiency of Lead. Annals of Occupational Hygiene, 59, 210-220.

[7] Rodrigues, E.G., Virji, M.A., McClean, M.D., Weinberg, J., Woskie, S. and Pepper, L.D. (2009) Personal Exposure, Behavior, and Work Site Conditions as Determinants of Blood Lead Among Bridge Painters. Journal of Occupational and Environmental Hygiene, 7, 80-87. https://doi.org/10.1080/15459620903418316

[8] Askin, D.P. and Volkmann, M. (1997) Effect of Personal Hygiene on Blood Lead Levels of Workers at a Lead Processing Facility. American Industrial Hygiene Association Journal, 58, 752-753. https://doi.org/10.1080/15428119791012414

[9] Nwudu, V., Fletcher, A.M. and Bauer, M. (2018) Patterns and Predictors of Personal Protection Compliance and Workplace Hygiene Behaviors among Workers with Elevated Blood Lead Levels in New York State. Journal of Occupational and Environmental Hygiene, 15, 654-663. https://doi.org/10.1080/15459624.2018.1487066

[10] Ulenbelt, P., Lumens, M., Géron, H., Herber, R., Broersen, S. and Zielhuis, R. (1990) Work Hygienic Behaviour as Modifier of the Lead Air-Lead Blood Relation. International Archives of Occupational \& Environmental Health, 62, 203. https://doi.org/10.1007/BF00379433

[11] Jackson, D.A. (2018) Notes from the Field: Lead Exposures among Employees at a Bullet Manufacturing Company-Missouri, 2017. Morbidity and Mortality Weekly Report, 67, 1103. https://doi.org/10.15585/mmwr.mm6739a7

[12] Virji, M.A., Woskie, S.R. and Pepper, L.D. (2008) Skin and Surface Lead Contamination, Hygiene Programs, and Work Practices of Bridge Surface Preparation and 
Painting Contractors. Journal of Occupational and Environmental Hygiene, 6, 131-142. https://doi.org/10.1080/15459620802656636

[13] ASTM International (2016) ASTM E1792-03: Standard Specification for Wipe Sampling Materials for Lead in Surface Dust. American Society for Testing and Materials, West Conshohocken.

[14] Esswein, E.J. and Ashley, K. (2003) Lead in Dust Wipes by Chemical Spot Test (Colorimetric Screening Method): Method 9105. In: Eller, P.M. and Cassinelli, M.E., Eds., NIOSH Manual of Analytical Methods, 4th Edition. https://www.cdc.gov/niosh/docs/2003-154/pdfs/9105.pdf

[15] Ignacio, J.S. and Bullock, W.H. (2006) A Strategy for Assessing and Managing Occupational Exposures. 3rd Edition, American Industrial Hygiene Association, Falls Church.

[16] Energy \& Building Research Center (EBRC), Kuwait Institute for Scientific Research (2007) Human Health Risk Assessment Guidance for Metals: Fact Sheet No. 1. https://www.ebrc.de/downloads/HERAG FS 01 August 07.pdf

[17] Multilingual IHSTAT+ [Computer Software] (2010). https://aiha-assets.sfo2.digitaloceanspaces.com/AIHA/resources/Public-Resources/ EASC-IHSTAT-V235.xls

[18] U.S. Environmental Protection Agency (EPA), Office of Pollution Prevention and Toxics (1994) Guidelines for Statistical Analysis of Occupational Exposure Data. https://www.epa.gov/tsca-screening-tools/guidelines-statistical-analysis-occupation al-exposure-data

[19] Boeniger, M. (2006) A Comparison of Surface Wipe Media for Sampling Lead on Hands. Journal of Occupational and Environmental Hygiene, 3, 428-434. https://doi.org/10.1080/15459620600802754

[20] Esswein, E.J. and Boeniger, M. (2005) Preventing the Toxic Hand Off. Occupational Hazards, 9, 53.

[21] Hewett, P. (2001) Industrial Hygiene Exposure Assessment-Data Analysis and Interpretation. In: Alaimo, R.J., Ed., Handbook of Chemical Health and Safety, Oxford University Press, Oxford, 102-129.

[22] Semple, S. (2004) Education: Dermal Exposure to Chemicals in the Workplace: Just How Important Is Skin Absorption? Occupational and Environmental Medicine, 61, 376. https://doi.org/10.1136/oem.2003.010645 